\title{
Salivary Antioxidant Capacity and Oral Health Status in Children with Visual Impairments-A Cross-Sectional Study
}

\author{
Madhurima Hazra ${ }^{1}$ Vabitha Shetty ${ }^{20}$ Lekshmi Radhakrishnan Suresh ${ }^{2}$ (1) \\ ${ }^{1}$ Clinical Practitioner, Dental Specialty Clinic, Ahmedabad, Gujarat, \\ Address for correspondence Vabitha Shetty, BDS, MDS, Department \\ India \\ 2 Department of Paediatric and Preventive Dentistry, AB Shetty \\ Memorial Institute of Dental Sciences, Nitte (Deemed to be \\ University), Karnataka, India \\ of Paediatric and Preventive Dentistry, AB Shetty Memorial Institute \\ of Dental Sciences, Nitte (Deemed to be University), Karnataka \\ 575018, India (e-mail: docvabitha29@gmail.com). \\ J Health Allied Sci ${ }^{\mathrm{NU}} 2022 ; 12: 322-329$.
}

\begin{abstract}
Keywords

- dental caries

- gingivitis

- free radicals

- antioxidants

- visual impairment
\end{abstract}

Background Children with visual impairment are at a higher risk for developing dental disease, and imbalances in the antioxidant levels may play an important role in the development and management of oral diseases.

Aim Our aim was to evaluate and compare the oral health status and salivary total antioxidant capacity (TAC) in children with and without visual impairment.

Methods Oral health was evaluated among 6 to 11 years old children with and without visual impairment (50 each) using World Health Organization oral health assessment form for caries prevalence, gingival health, and oral hygiene levels. Unstimulated saliva was collected and assayed for TAC using the phosphomolybdic method.

Statistical Analysis The data collected were tabulated, and the results, thus, obtained were subjected to statistical analysis using EZR software-version 1.37. The variables between the two groups were compared using the Mann-Whitney $U$ test and Fisher's exact test. A correlation test was done using Spearman's correlation test.

Results The mean TAC levels among children with and without visual impairment were $49.17( \pm 25.702)$ and $156.67( \pm 56.031) \mu \mathrm{g} / \mathrm{mL}$, respectively, which was statistically significant $(p<0.001)$. The children with visual impairment showed a significantly higher number of carious lesions compared with the control group $(p<0.001)$. Oral hygiene status and gingival health status were significantly poorer in children with visual impairments than the children in the control group $(p<0.001)$.

Conclusions Oral health was significantly poorer, and salivary TAC levels were significantly lower in children with visual impairment. There may be an inverse correlation between salivary TAC and oral health status. Clinical Significance: Since these children have been shown to have poorer oral health status, a baseline assessment of their salivary antioxidant capacity may indicate the need to reinforce oral care efforts and supplement them using an antioxidant-rich diet. published online January 18,2022
DOI https://doi.org/ $10.1055 / \mathrm{s}-0041-1741433$ ISSN 2582-4287.

\footnotetext{
(c) 2022. Nitte (Deemed to be University). All rights reserved.

This is an open access article published by Thieme under the terms of the Creative Commons Attribution-NonDerivative-NonCommercial-License, permitting copying and reproduction so long as the original work is given appropriate credit. Contents may not be used for commercial purposes, or adapted, remixed, transformed or built upon. (https://creativecommons.org/ licenses/by-nc-nd/4.0/) Thieme Medical and Scientific Publishers Pvt. Ltd., A-12, 2nd Floor, Sector 2, Noida-201301 UP, India
} 


\section{Background}

Oral health challenges for children with visual impairment are often complex, as they are frequently unable to apply techniques necessary for adequate plaque control. ${ }^{1}$ Children with visual impairments are at a disadvantage as far as maintenance of their oral health is concerned because early detection and recognition of oral diseases is a challenge for them. ${ }^{2}$ The individual's ability to address everyday tasks of personal hygiene, including oral hygiene, is critical in the maintenance of a healthy life.

It has been long recognized that saliva reflects the health of the individual as it contains proteins, hormones, antibodies, and other molecules that are frequently present in a person's blood and are used to monitor health and disease. ${ }^{3}$ Saliva contains antioxidants like albumin, ascorbic acid, glutathione, uric acid, and antioxidant enzyme systems that are derived from endogenous and exogenous sources. ${ }^{4,5}$ It can, thus, be measured for defense capacity using total antioxidant capacity (TAC), that is, the combined level of antioxidants available.

Antioxidants act by protecting the tissues from the potentially harmful effects of free radicals or reactive oxygen species (FR/ROS) formed from metabolic activities or chemical reactions that lead to excessive oxidation. ${ }^{6,7}$ These molecules are highly reactive and have been implicated in the literature to be major contributors to the pathogenesis of several systemic inflammatory diseases including oral pathologies such as dental caries and gingival and periodontal disease., ${ }^{8,9}$

Saliva is essentially the first line of defense against oxidative stress, and the salivary antioxidant system plays a major protective role against microorganisms, toxins, and harmful oxidation processes in the oral cavity. ${ }^{7}$ Recent advances in dental medicine have focused on two interrelated mecha- nisms by which this biological fluid and its antioxidant components assist in maintaining oral health in individua$1 \mathrm{~s}^{10}$ (- Fig. 1).

Since children with visual impairment are at a higher risk for developing dental disease, it is important to record and evaluate their oral health. Antioxidant levels may provide a clue into the long-term prognosis of the overall oral disease processes and the various health-promoting practices and behaviors developed in the children. To the best of our knowledge, no study has attempted to link TAC of saliva and oral health of children with visual impairment. Hence, there is a need for the present study.

Hence, the objectives of this study were to evaluate the oral health status and the TAC in saliva of children with visual impairments and to compare these levels against a suitable control group of children without visual impairments.

\section{Methods}

\section{Sample Size Estimation}

The sample size was estimated using the formula $\left[n=\left(Z_{\alpha / 2}+Z_{\beta}\right)^{2}{ }^{*}{ }^{*} \sigma^{2} / d^{2}\right]$, where $Z_{\alpha / 2}$ is the critical value of the normal distribution at $\alpha / 2, Z_{\beta}$ is the critical value of the normal distribution at $\beta, \sigma^{2}$ is the population variance, and $d$ is the effect size (derived from the previous study by Ahmadi-Motamayel et al). ${ }^{8}$ The calculation for the total number of samples in each group was, thus, determined to be 50 .

\section{Study Design}

\section{Inclusion Criteria}

Children diagnosed with visual impairment in the age group of 6 to 11 years were recruited from the government blind

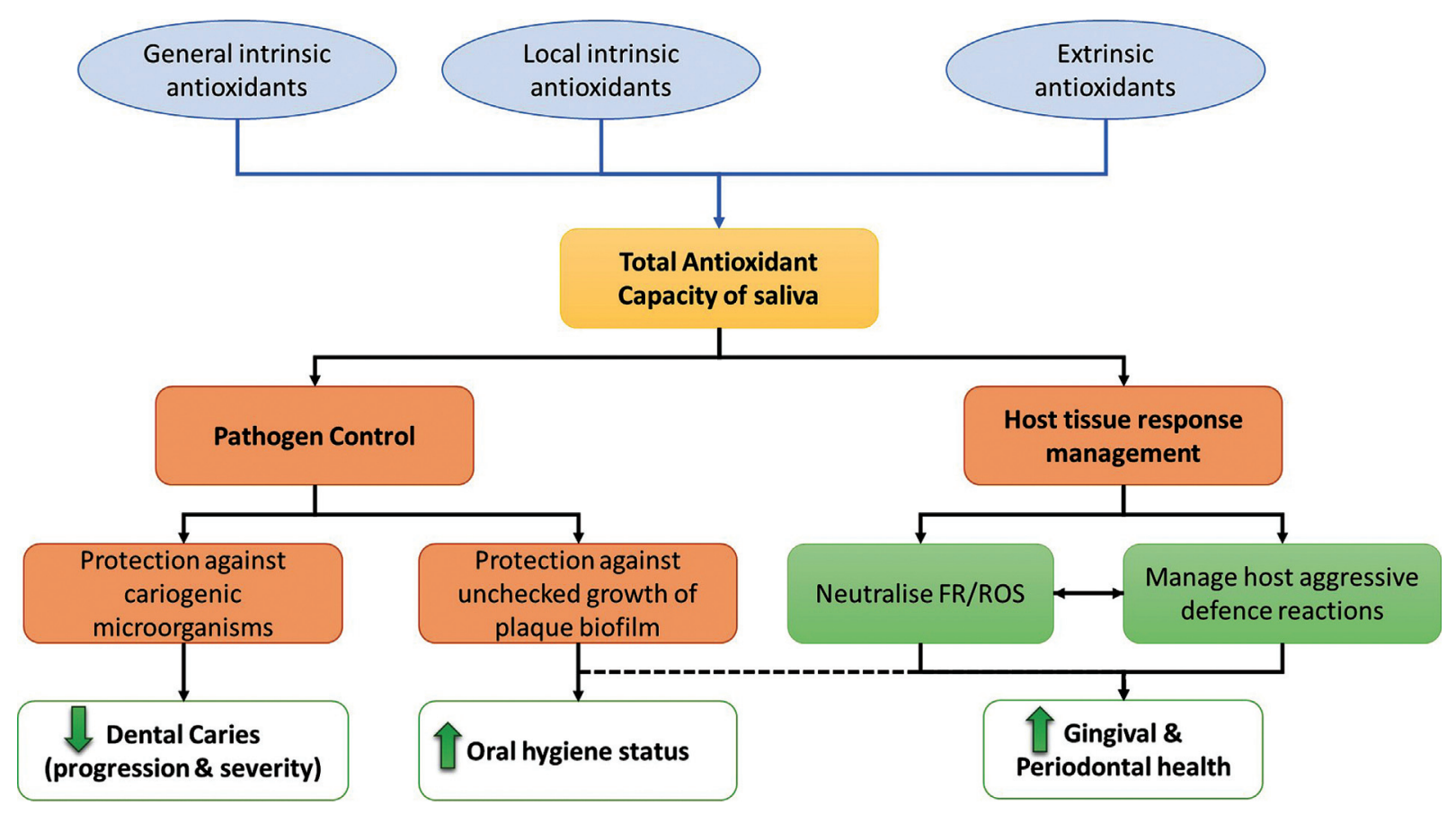

Fig. 1 Mechanism of action of TAC in the host oral defense. 
school, Kasaragod, Kerala. Children without visual impairment belonging to the age group of 6 to 11 years were randomly recruited from a government school in the same locality within the district. The nature of the study was explained to the potential participants and their parents/caretakers, following which written consent was obtained from the parents/caretakers and verbal assent was obtained from the participating children. The research was conducted in full accordance with the World Medical Association Declaration of Helsinki, and ethical clearance was granted by the Institutional Ethical Review Board (ABSM/EC/04/2010).

All children recruited in the study were administered a 3-day diet chart to assess their diet and dietary patterns. Fifty children were, thus, recruited in each group with similar diets and dietary patterns to minimize variability of dietary factors. The study group was designated as group 1 (children with visual impairment), and the control group was designated as group 2 (children without visual impairment).

\section{Exclusion Criteria}

The exclusion criteria were as follows:

- children with partial visual impairment,

- children with other handicapping conditions/illnesses,

- children with chronic/systemic disease, and

- children on medication.

\section{Method of Collection of Data}

Recording the Oral Health Status-Oral health status was evaluated using the standardized World Health Organization Oral Health Assessment Form for children (2013). ${ }^{11}$ The oral health examination of children included assessment of caries experience using the decayed (d/D), exfoliated, extracted or missing (e/M), and filled (f/F) (deft/DMFT) indices. Gingival health was recorded using the gingival index $(\mathrm{GI}){ }^{12}$ and oral hygiene level was assessed using the Simplified Oral Hygiene Index (OHI-S). ${ }^{13}$

Children were seated on an ordinary chair at their respective schools/centers, and their oral cavities were examined under good illumination (either natural light or hand torch), using a sterile mouth mirror and Community Periodontal Index (CPI) probe. $^{11,14}$ Protective cross-infection protocol was observed using disposable hand gloves and masks. All examinations were conducted by a single examiner.

Saliva Collection-Children were instructed not to eat, drink, or perform any oral hygiene practices at least 2 hours before collection time. The salivary samples were collected from the children at daytime, between 11:30 Am and 12 noon. The sterile cotton roll was placed sublingually, and the saliva was allowed to accumulate in the floor of the mouth for 2 minutes. The cotton rolls, with the adsorbed, unstimulated, whole saliva, were then placed inside a $15 \mathrm{~mL}$ sterile plastic tube with a sterile $100 \mathrm{~mL}$ pipette tip in the bottom to facilitate saliva collection by centrifugation at $10,000 \mathrm{~g}$ for 10 minutes and stored at a temperature of $4^{\circ} \mathrm{C} .{ }^{15}$ The collected saliva was subjected to TAC analysis using the phosphomolybdic acid method. ${ }^{16}$

\section{Estimation of Total Antioxidant Capacity of Saliva}

The phosphomolybdic acid method is a quantitative assay based on the conversion of molybdenum (Mo.VI) by reducing agents like antioxidants to molybdenum (Mo.V), which further reacts with phosphate under acidic $\mathrm{pH}$ resulting in the formation of a green-colored complex, the intensity of which can be read spectrophotometrically at $695 \mathrm{~nm}$.

A clean test tube is used to pipette out $100 \mu \mathrm{L}$ of the sample (saliva). To precipitate out the proteins in the salivary samples, $100 \mu \mathrm{L}$ of $5 \% \mathrm{TCA}$ is then added and centrifugation of the mixture is then done after allowing it to stand for approximately 5 minutes.

$100 \mu \mathrm{L}$ of the clear supernatant obtained (free of protein precipitates) is then transferred into a clean test tube and $1 \mathrm{~mL}$ of TAC reagent [0.6 Mm conc. sulfuric acid $\left(\mathrm{H}_{2} \mathrm{SO}_{4}\right)+28$ Mm sodium dihydrogen phosphate $\left(\mathrm{Na} \mathrm{H}_{2} \mathrm{PO}_{4}\right)+4 \mathrm{mM}$ ammonium heptamolybdate $\left.\left(\mathrm{NH}_{4}\right)_{6} \mathrm{Mo}_{7} \mathrm{O}_{24} \cdot 4 \mathrm{H}_{2} \mathrm{O}\right)+$ ascorbic acid (vitamin c)] is added to it and incubated in water bath at $90^{\circ} \mathrm{C}$ for 90 minutes. $100 \mu \mathrm{L}$ of water is substituted instead of saliva in the reaction mixture which acts as blank. After the incubation, the reaction mixture is allowed to cool, and the optical density of the greenish to bluish color formed is read at $695 \mathrm{~nm}$ against blank.

\section{Statistical Analysis}

The data collected were tabulated, and the results, thus, obtained were subjected to statistical analysis using EZR software-version $1.37 .^{17}$ Significance of $p$-value was set to $95 \%$ confidence interval $(\alpha=0.05)$ and $80 \%$ power $(\beta)$. The assumption of normalcy was ruled out using Shapiro-Wilk test, prior to the decision to use non-parametric tests for intergroup comparisons. The numbers of carious lesions and the TAC of saliva between the children of both groups were compared using Mann-Whitney U test. The distribution of children according to their gingival health and oral hygiene statuses was compared between the two groups using Fisher's exact test. Tests for correlation between TAC and the oral health variables were done using Spearman's rho $(\rho)$ correlation analysis.

\section{Results}

Demographics-All children included in the study were between the ages of 6 and 11 years, with a mean age of 9.7 $( \pm 2.005)$ years in group 1 and $8.2( \pm 1.617)$ years in group 2 . There were 26 boys and 24 girls in group 1 and 23 boys and 27 girls in group 2.

Salivary TAC-The mean and median TAC levels among children with visual impairment were $49.17( \pm 25.702)$ and $48.55 \mu \mathrm{g} / \mathrm{mL}$, whereas in children without visual impairments the levels were $156.67( \pm 56.031)$ and $153.80 \mu \mathrm{g} / \mathrm{mL}$, respectively. The difference between the median TAC levels in the two groups was statistically significant $(p<0.001)$ (-Table 1).

Caries Experience-The mean deft and DMFT values in group 1 children were $0.92( \pm 1.747)$ and $2.44( \pm 1.786)$, while the corresponding mean deft and DMFT values for children in group 2 were $0.2( \pm 0.606)$ and $0.12( \pm 0.385)$. The 
Table 1 Comparison of salivary TAC levels $(\mu \mathrm{g} / \mathrm{mL})$ and caries status of the children in the two groups

\begin{tabular}{|c|c|c|c|c|c|c|}
\hline \multicolumn{7}{|c|}{ Salivary TAC levels $(\mu \mathrm{g} / \mathrm{mL})$} \\
\hline & $n$ & Min & $\begin{array}{l}\text { Median } \\
(\mathrm{Q} 1, \mathrm{Q} 3)\end{array}$ & Max & $w$ & $p$-Value ${ }^{a}$ \\
\hline Group 1 & 50 & 5.8 & $\begin{array}{l}48.55 \\
(27.8,70.3)\end{array}$ & 95.3 & \multirow[t]{2}{*}{2.5} & \multirow[t]{2}{*}{$8.18 e-18$} \\
\hline Group 2 & 50 & 93.8 & $\begin{array}{l}153.80 \\
(120.55,165.17)\end{array}$ & 406.3 & & \\
\hline \multicolumn{7}{|c|}{ Caries lesions } \\
\hline & $n$ & Min & $\begin{array}{l}\text { Median } \\
(\mathrm{Q} 1, \mathrm{Q} 3)\end{array}$ & Max & $w$ & $p$-Value ${ }^{a}$ \\
\hline Group 1 & 50 & 0 & $3(2,4)$ & 12 & \multirow[t]{2}{*}{2,244} & \multirow[t]{2}{*}{$3.99 e-13$} \\
\hline Group 2 & 50 & 0 & $0(0,0)$ & 4 & & \\
\hline
\end{tabular}

Abbreviation: TAC, total antioxidant capacity.

${ }^{a} p>0.05$ not significant; $p<0.05$ significant.

contributing component among the decayed (d/D), exfoliated, extracted or missing (e/M), and filled ( $\mathrm{f} / \mathrm{F})$ criteria in the deft and DMFT indices for both groups of children was that for decay (d/D). Collectively, in terms of caries experience, the mean and median number of carious lesions in group 1 and group 2 were $3.36( \pm 2.585)$ and 3 , and $0.32( \pm 0.819)$ and 0 , respectively. When the median of carious lesions in the two groups was compared, there was a statistically significant difference $(p<0.001)$ ( - Table 1 ).

Oral Hygiene Status-There was a statistically significant difference between the corresponding percentages of children exhibiting good, fair, and poor oral hygiene when compared among the children of the two groups $(p<0.001)$ (-Table 2$)$.

Gingival Status-There was a statistically significant difference between the corresponding percentages of children exhibiting mild, moderate, and severe gingivitis between the children of the two groups ( $p<0.001)$ (-Table 2 ).

Correlating Caries Experience, $\mathrm{OHI}-\mathrm{S}$, and Gingival Status with TAC-There was an inverse correlation observed between the number of carious lesions, OHI-S, and GI scores with TAC values in the children with visual impairment, which had a strong correlation and was statistically significant $(p<0.001)$ ( - Table 3). Similarly, there was an inverse correlation observed between the number of carious lesions, OHI-S, and gingival scores with TAC values of saliva in the control group of children, which also had a strong correlation and was statistically significant $(p<0.001)$ (-Table 3 ). Thus, we observed that as the number of carious lesions, OHI-S scores, and GI scores increased, the TAC of their saliva decreased and vice versa (-Fig. 2).

Table 2 Comparison of the distribution of children according to their OHI-S and gingival index scores in the two groups

\begin{tabular}{|c|c|c|c|c|c|}
\hline \multicolumn{6}{|l|}{ OHI-S scores } \\
\hline $\begin{array}{l}\text { Oral hygiene status } \\
\text { (score interpretation) }\end{array}$ & Score range & Total & Group 1 & Group 2 & 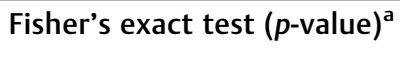 \\
\hline Good & $0.0-1.2$ & 51 & 1 & 50 & \multirow[t]{4}{*}{$1.01 e-27$} \\
\hline Fair & $1.3-2.9$ & 30 & 30 & 0 & \\
\hline \multirow[t]{2}{*}{ Poor } & $3.0-6.0$ & 19 & 19 & 0 & \\
\hline & Total & 100 & 50 & 50 & \\
\hline \multicolumn{6}{|l|}{ Gingival index scores } \\
\hline $\begin{array}{l}\text { Gingival status } \\
\text { (score interpretation) }\end{array}$ & Score range & Total & Group 1 & Group 2 & Fisher's exact test ( $p$-value $)^{a}$ \\
\hline Normal & 0 & 2 & 0 & 2 & \multirow[t]{5}{*}{$4.55 e-25$} \\
\hline Mild inflammation & $0.1-1.0$ & 47 & 1 & 48 & \\
\hline Moderate inflammation & $1.1-2.0$ & 42 & 40 & 2 & \\
\hline \multirow[t]{2}{*}{ Severe inflammation } & $2.1-3.0$ & 9 & 9 & 0 & \\
\hline & Total & 100 & 50 & 50 & \\
\hline
\end{tabular}

Abbreviation: OHI-S, Oral Hygiene Index-simplified scores.

${ }^{a} p>0.05$ not significant; $p<0.05$ significant. 
Table 3 Correlation between salivary TAC levels $(\mu \mathrm{g} / \mathrm{mL})$ and variables on oral health

\begin{tabular}{|l|l|l|l|l|l|}
\hline \multicolumn{2}{|l|}{ Spearman's rank correlation test } & Caries lesions & OHI-S & Gingival index \\
\hline \multirow{2}{*}{ Group 1 } & \multirow{2}{*}{ TAC levels } & $\rho^{\mathrm{a}}$ & -0.475 & -0.999 & -0.992 \\
\cline { 3 - 6 } & & $p$-value $^{\mathrm{b}}$ & $4.86 \mathrm{e}-4$ & $8.28 \mathrm{e}-63$ & $1.3 \mathrm{e}-44$ \\
\cline { 3 - 6 } & & $N$ & 50 & 50 & 50 \\
\hline \multirow{2}{*}{ Group 2 } & \multirow{2}{*}{ TAC levels } & $\rho^{\mathrm{a}}$ & -0.562 & -0.873 & -0.909 \\
\cline { 3 - 6 } & & $p$-value ${ }^{\mathrm{b}}$ & $2.15 \mathrm{e}-5$ & $1.5 \mathrm{e}-16$ & $8.12 \mathrm{e}-20$ \\
\cline { 3 - 6 } & & $N$ & 50 & 50 & 50 \\
\hline
\end{tabular}

Abbreviations: OHI-S, Oral Hygiene Index-simplified scores; TAC, total antioxidant capacity.

${ }^{a} \rho$ value: $0.01-0.29$ weak relationship; $0.3-0.39$ moderate relationship; $0.40-0.69$ strong relationship; and $\geq 0.70$ very strong relationship.

b $p>0.05$ not significant; $p<0.05$ significant.

\section{Discussion}

An FR/ROS may be defined as "an atomic or molecular species with one or more unpaired electrons in its structure." ${ }^{18}$ This impaired electron destabilizes the molecules, leading to oxidative stress. The body of an organism is equipped with certain scavengers and reparatory processes to stabilize these FR/ROS molecules, thereby reducing the resultant stress that accompanies them. Oxidative stress is, thus, an imbalance between FR/ROS molecules and the antioxidant system. This imbalance has been implicated as an important contributory factor in the pathology of several oral diseases. ${ }^{19-21}$

The antioxidant system in humans constitutes a highly complex system of molecules and chemicals that interplay to form an effective network of defense against the disease process. ${ }^{18,22}$ Hence, the evaluation of antioxidants may give an indication of the susceptibility of the individual to various oral diseases. While it is important to evaluate the amount and activity of each individual antioxidant system, recent literature has directed focus toward evaluating the collective capacity of these systems in the form of 'TAC' of biological fluids, including blood and saliva.

Blood is usually the gold standard in laboratory tests to monitor health and disease, but saliva is easier to collect, less invasive, and is, therefore, especially convenient for use in children. It may also act as a better indicator of local disease processes like dental caries and gingival/periodontal pathologies. Additionally, since nearly all the analytes that are present in blood are also expressed in saliva, the latter may be used as a suitable alternative. ${ }^{23}$
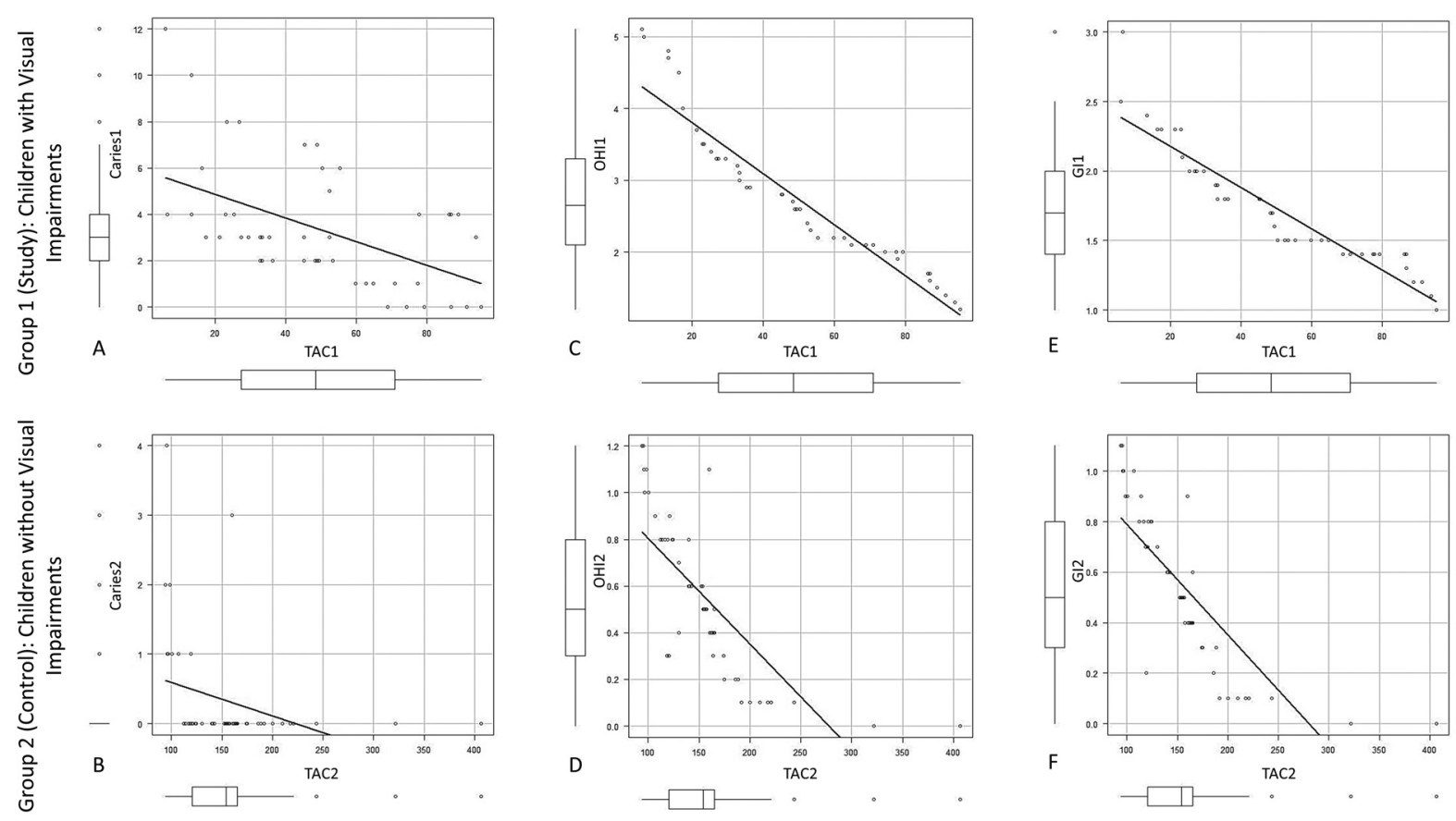

Fig. 2 Depicts the relationship of the respective salivary TAC levels when correlated with (A, B) caries status, (C, D) oral hygiene index scores, and $(E, F)$ gingival index scores among the children in (A, C, E) the study group and (B, D, F) the control group. The abbreviations TAC, Caries, OHI$\mathrm{S}$, and $\mathrm{Gl}$ on the graphs relate to total antioxidant capacity, caries prevalence, Oral Hygiene Index-simplified scores, and gingival index scores, respectively. 
There is evidence in the literature to suggest that among the various forms of saliva, unstimulated whole saliva, collected passively from the floor of the mouth, is a better, more accurate fluid for the assessment of the resting conditions in the human body. As TAC is higher in unstimulated saliva, these samples have been preferred in the determination of antioxidant defense parameters in the oral cavity. ${ }^{23}$

We examined a total of 100 children, aged between 6 and 11 years, for their salivary TAC estimates -50 children with visual impairment (study group) and 50 children without visual impairment (control group). Since dental caries is multifactorial and dietary factors play a major role in caries and antioxidant level estimations, it was decided to keep geographical and dietary variables constant between the two groups of children. Despite this, however, the caries experience of children with visual impairment was significantly higher when compared with those of the control group. The mean DMFT among the children with visual impairment was found to be 2.44 , which is comparable to the results obtained by Tamagond et al, who found a significantly higher caries score in non-sighted children as compared with sighted children. ${ }^{24}$ The increased caries experience among children with visual impairment in the present study may be attributed to their inadequate levels of oral hygiene practices when compared with the control group.

TAC of saliva ranged between 5.8 and $95.3 \mu \mathrm{g} / \mathrm{mL}$ in children with visual impairment. Interestingly, these values were similar to those obtained by Rai et al in children with autism. ${ }^{25}$ However, we observed comparatively higher TAC levels in our control group of children $(93.8-406.3 \mu \mathrm{g} / \mathrm{mL}$ ) than those of the control group of their study, which comprised of non-autistic siblings. ${ }^{25}$ This raises the possibility that there could be predetermined genetic factors which may influence the production of antioxidants in children.

In children with visual impairment, when the relation between caries and TAC was analyzed, we observed that there was an inverse correlation with increasing caries experience. In the control group, a similar inverse correlation was observed between caries experience and TAC levels (-Fig. 2). These findings agree with the results of previous studies that reported an increase in salivary TAC levels in children with an increasing number of carious lesions. ${ }^{26,27}$

According to Krawczyk et al, the presence of cariogenic bacteria draws "an enhanced response of leukocytes, like neutrophils and monocytes, which generate ROS in a respiratory burst process, causing a bactericidal effect."27 This might explain the decrease in TAC in the saliva of children with an increased prevalence of dental caries. An increase in the levels of salivary ROS intensifies the oxidative stress as a result of the reduction in the TAC of saliva, and maybe even vice-versa. Thus, the low TAC values in children with visual impairment may be attributed to a higher infectious challenge in their oral cavities. However, other studies have contradicted our findings and have found an increase in salivary TAC levels in children with an increasing prevalence of dental caries. . $^{8}$

In our study when the oral hygiene status was assessed, it was observed that the majority of the children with visual impairment exhibited fair to poor oral hygiene. This could be attributed to the decreased ability of these children in maintaining their oral hygiene, caused by the lack of manual-visual coordination. These findings are in accordance with the results of a previous study, where the OHI-S score for the individuals with visual impairment indicated that they had mostly fair oral hygiene. ${ }^{29}$ When the relation between oral hygiene status and TAC was analyzed in the children with visual impairment, we observed that there was an inverse correlation, between the two variables (-Fig. 2). This is similar to the study done by Krawczyk et al, where they found a negative correlation between $\mathrm{OHI}-\mathrm{S}$ and TAC in adolescents. In the control group, all children were observed to have good oral hygiene status. ${ }^{27}$ This could be attributed to the school dental health programs made available to these children and the awareness of their parents regarding the importance of regular dental check-ups, which occurs less in children with visual impairments. These children were also observed to have increased TAC levels that show an inverse relationship between OHI-S scores and TAC levels (-Fig. 2).

In our study, we found that majority of the children with visual impairment had moderate to severe gingivitis, which indicates the presence of significant gingival inflammation in these children. These findings are in accordance with the results of Liu et al who found poor periodontal health in a group of visually impaired schoolchildren in Northeast China. ${ }^{30}$ Further, in these children when the relation between gingival scores and TAC was analyzed, we observed that there was an inverse correlation, i.e., with increasing scores on GI, TAC levels decreased (-Fig. 2). Our findings are consistent with the results of a study by Sculley and Langley-Evans who found that periodontal disease is associated with reduced salivary antioxidant status and increased oxidative damage within the oral cavity. ${ }^{19}$ The presence of gingivitis itself is reported to cause an increased production of FR species. ${ }^{31}$ We surmise that the poorer oral health status of the children with visual impairment could have depleted the salivary antioxidant levels. Meanwhile, most of the children in the control group exhibited only mild gingivitis. In this group, a similar inverse correlation was observed between GI scores and TAC levels (-Fig. 2).

If salivary TAC levels increase with increasing levels of oral pathologies like dental caries, it stands to reason that, being protective in its role, TAC levels may rise in the oral cavity to combat the oxidative stress produced by the disease processes in the oral environment. However, our study shows that there is a clear association between decreasing salivary TAC levels and increasing levels of oral pathologic conditions like dental caries, poor gingival health, and declining oral hygiene status. Might this observation be rationalized with the reasoning that reduced levels of salivary TAC indicate the depletion of these defensive molecules by the local disease processes, followed by a probable failure to be supplemented by the body's endogenous production mechanisms or dietary supplementation. 


\section{Limitations and Future Scope}

In our study, children in the study group were recruited from a government school which catered solely to the educational needs of children with visual impairments. This meant that an in-house group of controls, which would have been more desirable, were unavailable as controls in the study. Children in the control group were, thus, recruited from a neighboring school to avoid variations in socioeconomic and regional factors.

Another possible limitation is that, while our study helps establish the association of salivary TAC in the oral disease process being more critical among children with visual impairments, it does not throw light on its causation. There is, thus, a need to investigate the exact mechanism and role of TAC in the oral disease process. Further studies in this direction should be corroborated using longitudinal estimates to assess the potential use of salivary antioxidants to combat oral pathologies and its role as a prognostic marker for disease severity. There is also a need to investigate and establish effective amounts of antioxidants needed for optimal oral health among various populations of children, including those with special health care needs.

\section{Conclusions}

Overall, the oral health status of the children with visual impairment was significantly poorer as compared with the children without visual impairment. Salivary TAC was significantly reduced in children with visual impairment, and TAC levels in all children seemed to have an inverse correlation with caries experience, oral hygiene, and gingival status. As children with visual impairment are at a higher risk for oral diseases, we may improve the antioxidant defense capacity of these children by including a diet rich in antioxidants. However, further research should be directed toward assessing the dental needs of children with visual impairment and other handicapping conditions, while understanding the interplay of various predisposing and health-promoting factors, such as salivary TAC.

\section{Funding}

This study was done as part of the short-term student research program and was funded by the Indian Council of Medical Research (ICMR) [2010-01171].

\section{Conflict of Interest}

None declared.

\section{Acknowledgments}

The funding organization was not involved in the study design, data collection, storage and management, data analysis and interpretation, writing of the report, or decision to submit for publication.

\section{References}

1 Schembri A, Fiske J. The implications of visual impairment in an elderly population in recognizing oral disease and maintaining oral health. Spec Care Dentist 2001;21(06):222-226

2 Rao D, Hegde A, Munshi AK. Oral hygiene status of disabled children and adolescents attending special schools of South Canara, India. Hong Kong Dent J 2005;2:107-110

3 Malathi L, Rajesh E, Babu NA, Jimson S. Saliva as a diagnostic tool. Biomed Pharmacol J 2016;9(02):867-870

4 Miricescu D, Greabu M, Totan A, Didilescu A, Rădulescu R. The antioxidant potential of saliva: clinical significance in oral diseases. Ther Pharmacol Clin Toxicol 2011;15(02):139-143

5 Minic I. Antioxidant role of saliva. J Otolaryngol Res 2019;2:124

6 O'Brien PJ. Antioxidants and cancer: molecular mechanisms. In: Free Radicals in Diagnostic Medicine. Boston, MA: Springer; 1994

7 Battino M, Ferreiro MS, Gallardo I, Newman HN, Bullon P. The antioxidant capacity of saliva. J Clin Periodontol 2002;29(03): 189-194

8 Ahmadi-Motamayel F, Goodarzi MT, Hendi SS, Kasraei S, Moghimbeigi A. Total antioxidant capacity of saliva and dental caries. Med Oral Patol Oral Cir Bucal 2013;18(04):e553-e556

9 Miricescu D, Totan A, Calenic B, et al. Salivary biomarkers: relationship between oxidative stress and alveolar bone loss in chronic periodontitis. Acta Odontol Scand 2014;72(01):42-47

10 Salivary Antioxidants Accessed April 13, 2021 at: https:// dentalantioxidants.com/salivary-antioxidants

11 WHO. Oral Health Surveys, Basic Methods. 5th ed. Geneva: World Health Organization; 2013 Accessed April 13, 2021 at: https:// www.who.int/oral_health/publications/9789241548649/en/

12 Löe $\mathrm{H}$. The gingival index, plaque index and the retention index systems. J Periodontol 1967;38(06):610-616

13 Greene JC, Vermillion JR. The simplified oral hygiene index. J Am Dent Assoc 1964;68:7-13

14 Ishizuka Y, Maki Y, Kagami N, Satou R, Sugihara N. Comparison of dental explorers and CPI-probes in diagnosing dental caries. Bull Tokyo Dent Coll 2015;56(03):139-144

15 Henson BS, Wong DT. Collection, storage, and processing of saliva samples for downstream molecular applications. In: Seymour G, Cullinan M, Heng N, eds. Oral Biology. Methods in Molecular Biology (Methods and Protocols). Totowa, NJ: Humana Press; 2010666:21-30

16 Prieto P, Pineda M, Aguilar M. Spectrophotometric quantitation of antioxidant capacity through the formation of a phosphomolybdenum complex: specific application to the determination of vitamin E. Anal Biochem 1999;269(02):337-341

17 Kanda Y. Investigation of the freely available easy-to-use software 'EZR' for medical statistics. Bone Marrow Transplant 2013;48(03): $452-458$

18 Battino M, Bullon P, Wilson M, Newman H. Oxidative injury and inflammatory periodontal diseases: the challenge of anti-oxidants to free radicals and reactive oxygen species. Crit Rev Oral Biol Med 1999;10(04):458-476

19 Sculley DV, Langley-Evans SC. Salivary antioxidants and periodontal disease status. Proc Nutr Soc 2002;61(01):137-143

20 Akalin FA, Baltacioğlu E, Alver A, Karabulut E. Lipid peroxidation levels and total oxidant status in serum, saliva and gingival crevicular fluid in patients with chronic periodontitis. J Clin Periodontol 2007;34(07):558-565

21 Canakci CF, Cicek Y, Yildirim A, Sezer U, Canakci V. Increased levels of 8-hydroxydeoxyguanosine and malondialdehyde and its relationship with antioxidant enzymes in saliva of periodontitis patients. Eur J Dent 2009;3(02):100-106

22 Chapple IL. Reactive oxygen species and antioxidants in inflammatory diseases. J Clin Periodontol 1997;24(05):287-296

23 Edgar WM. Saliva: its secretion, composition and functions. $\mathrm{Br}$ Dent J 1992;172(08):305-312 
24 Tamagond S, Zaparde N, Gunda S, Patil A, Sandhyarani B. Comparison of oral health status, oral health knowledge and oral hygiene practices in non-sighted and sighted institutionalized children of Sangli. Int J Curr Res 2018;10(03): 66588-66591

25 Rai K, Hegde AM, Jose N. Salivary antioxidants and oral health in children with autism. Arch Oral Biol 2012;57(08):1116-1120

26 Rahmani M, Ghorchi V, Rezaei F, Vaisi-Raygani A. Evaluation of total antioxidant capacity of saliva in high school students. Glob J Health Sci 2015;8(04):89-94

27 Krawczyk D, Blaszczak J, Borowicz J, Mielnik - Blaszczak M. Lifestyle and risk of development of dental caries in a population of adolescents. Ann Agric Environ Med 2014;21:576-580
28 Dodwad R, Betigeri AV, Preeti BP. Estimation of total antioxidant capacity levels in saliva of caries-free and caries-active children. Contemp Clin Dent 2011;2(01):17-20

29 John JR, Daniel B, Paneerselvam D, Rajendran G. Prevalence of dental caries, oral hygiene knowledge, status and practices among visually impaired individuals in Chennai, Tamil Nadu. Int J Dent 2017;2017:9419648

30 Liu L, Zhang Y, Wu W, et al. Oral health status among visually impaired school children in Northeast China. BMC Oral Health 2019;19(01):63

31 Chapple ILC. Role of free radicals and antioxidants in the pathogenesis of the inflammatory periodontal diseases. Clin Mol Pathol 1996;49(05):M247-M255 